\title{
Group Coupon Recommendation System for Mobile Users
}

\author{
M. Thenmozhi \\ Assistant Professor, \\ Department of Computer \\ Science and Engineering, \\ Pondicherry Engineering College, India
}

\author{
T. P. Ezhilarasi \\ P.G Student, \\ Department of Computer \\ Science and Engineering, \\ Pondicherry Engineering College, India
}

\begin{abstract}
Recommendation systems have become an essential component of online marketing. Group recommendation is a challenging issue due to the diversity and dynamics involved in the groups. The existing works in group recommendation mainly focused on content interest of group members ignoring other characteristic useful for improving the recommendations. In this paper, a group-coupon recommendation system has been proposed. It recommends location sensitive products to customers and helps them to form groups in order to avail the discount provided by the sellers in a group purchase. As the usage of smart phones has increased, a mobile application called promoterApp has been developed based on the proposed recommendation approach. Experiments were conducted for WhatsApp users to recommend group discounts.
\end{abstract}

\section{Keywords}

Recommendation System, Group Purchase, Location Sensitive Service.

\section{INTRODUCTION}

Recommender system is a specific type of information filtering technique which is used to recommend commercial items that could be interesting to a specific user [6]. These systems have become extremely common in recent years, and are applied in a variety of applications. The most popular ones are movies, music, news, books, research articles, search queries, social tags, and products in general. However, there are also recommender systems for experts, jokes, restaurants, financial services, life insurance, persons (online dating), and Twitter followers.

Adomavicius classified recommendation methods into three categories: content-based, collaborative, and hybrid recommendation algorithms [8]. Content-based methods extract the appropriate content for the user based on similarity to the contents indicated by user preference. Collaborative methods recommend the content highly rated by other users who share the target user's preferences. Hybrid methods combine content-based and collaborative methods. These recommendation methods attempt to identify what the user is expected to find interesting.

Though most recommendation systems make suggestions for individual users in certain circumstances the suggested items are needed for a group of users. For example, a group of friends or a family is planning to watch a movie. In case of such group-buying commerce, the sellers provide products or services at significantly discounted prices online on the condition that there exists a minimum number of buyers willing to buy the same item [7]. Although customers are certainly attracted to the products offered at a substantial discount they may not have the patience to put in the effort in forming a consumer group. Though there have been attempts to establish group recommendations they mainly focus on offline environment. In this paper a recommendation approach has been proposed which recommends location sensitive products to the customers and helps to form a group and get the maximum discount for the products.

\section{RELATED WORK}

In [1] the authors proposed a system which is designed based on the framework of collaborative filtering. Especially, they used genetic algorithm to predict the possible interactions among group members. With this they could estimate the rating given by a group of members for a particular item. In [2] they proposed an improved group recommendation procedure which could improve the group recommendation effectiveness and the satisfaction of individual group members. It consists of two phases where, the first phase was to generate a recommendation set for a group using the collaborative filtering method which is used by existing group recommendation systems. And the second phase was to remove irrelevant items from the recommendation which could improve the satisfaction of individual member's preference. In [3] the authors proposed a map-based personalized recommendation system. It reflects the user's preference modeled by Bayesian Networks (BN). Here the structure of the $\mathrm{BN}$ is built by an expert while the parameter is learned from the data sets. The proposed system collects contextual information such as location, time, weather, and user request from the mobile device. It infers the most preferred item and provides an appropriate service by displaying it on the mini map. In [4] they introduced GroupReM which makes movie recommendations that are interesting to members of a group by (i) employing a merging strategy to explore the interests of individual, group members in movies and create a profile that reflects the movie preferences of the group, (ii) using word correlation factors to find movies similar in content, and (iii) considering the popularity of movies at a movie website. In [5] the authors introduced group recommendation system (GRS) using a combination of hierarchical clustering technique and decision tree. They believed that Facebook social network groups can be identified based on their members' profiles. In [9] the authors proposed gCOACH (COllaborative Advisory Channel) for group recommendations. They have provided a web-based environment for enabling the interaction of users from any location with the help of case-based group recommender. In [10] they used a hybrid technique that combines collaborative, content-based and demographic filtering to provide group recommendation for tourists. Their approach was materialized in a recommender system named Hermes. It suggests tourist attractions to both individuals and 
groups of users. Though in literature group recommendation systems have been proposed, they do not much focus on mobile commerce. In Table 1 a comparison of the existing works in group recommendation has been provided. As people have moved to smart phones it is necessary to provide a mobile application that can recommend products with discounts when brought in groups. Hence a new methodology for recommending items along with group coupon has been proposed in this paper.

Table 1. Comparison of existing works

\begin{tabular}{|c|l|l|}
\hline Existing Work & \multicolumn{1}{|c|}{ Technique Used } & \multicolumn{1}{|c|}{ Limitations } \\
\hline$[3]$ & $\begin{array}{l}\text { Bayesian Networks and parameter } \\
\text { learning technique }\end{array}$ & $\begin{array}{l}\text { Bayesian networks built by an expert cannot } \\
\text { reflect a change of environment. }\end{array}$ \\
\hline$[2]$ & Collaborative filtering method & $\begin{array}{l}\text { Dissatisfaction of a small number of group } \\
\text { members while satisfying the majority. }\end{array}$ \\
\hline$[1]$ & $\begin{array}{l}\text { Collaborative filtering method } \\
\text { and genetic algorithm }\end{array}$ & $\begin{array}{l}\text { Not suitable for supporting group purchasing } \\
\text { decisions. }\end{array}$ \\
\hline$[4]$ & $\begin{array}{l}\text { Content-based strategy using rank } \\
\text { aggregation model }\end{array}$ & $\begin{array}{l}\text { No consideration on dynamic preferences of } \\
\text { group members. }\end{array}$ \\
\hline$[5]$ & $\begin{array}{l}\text { Clustering technique and decision } \\
\text { tree }\end{array}$ & Location sensitive groups are not formed. \\
\hline$[9]$ & Case based group recommender & Not suitable for mobile users. \\
\hline$[10]$ & $\begin{array}{l}\text { Hybrid Filtering for group } \\
\text { recommendation }\end{array}$ & Suitable only for tourism recommendation \\
\hline
\end{tabular}

\section{GROUP COUPON RECOMMENDATION SYSTEM}

\subsection{Overview of the Proposed Approach}

The proposed approach is mainly focused on recommending location sensitive products for a particular customer. Customers' decision making with regard to purchasing in a physical store was analyzed based on three main factors: the similarity between user preference and product characteristics, the geographic convenience of store position, and the influence of friends' evaluation of the product. In order to use the proposed recommendation system in mobile devices a mobile application called promoterApp has been developed for a group-discount recommendation mechanism.

The existing approaches work only for an individual recommendation and do not encourage group purchase. Hence, the proposed system brings benefits to both the trader by selling a number of products in the name of group discount and the customer by getting the product at maximum discount.

The local shop is benefited by this promoterApp. That is, when the local shop traders register in this application, their location is obtained by GPS and stored. They can post products available in their shops and also can view the details of the customer who have purchased in their shops through this application. They are also provided with the transaction details for the product they have sold. The trader is provided with a QR code scanner with which they can scan the code and offer the discount for the customer.
The products are recommended to the customer with respect to the nearby located shops. When the customer logs in, the top purchased products are listed for the customers and if the user wishes to look the top rated products he/she has to use sort by rating, then the products are sorted according to ratings. The user can share the products in order to increase his discount. When his friend buys the shared product the user will be updated with new discount coupon. The user can get the maximum discount when the minimum number of people mentioned for that product has purchased. When a customer buys a product few shops are listed with respect to the customer's location. When a shop is selected by the customer then a QR code is generated for the product and sent to the customer email address. The customer can provide this $\mathrm{QR}$ code in the respective shops and get maximum discount for his product.

\subsection{Working Model}

The proposed promoterApp uses a combination of several techniques. These techniques are used for producing best recommendation of the products and services to the users. The workflow with respect to customer view is presented in Fig 1. The workflow with respect to trader view is presented in Fig. 2. This application takes user ratings and location of the customer as input. The components involved in the proposed approach for the promoterApp have been explained below. 


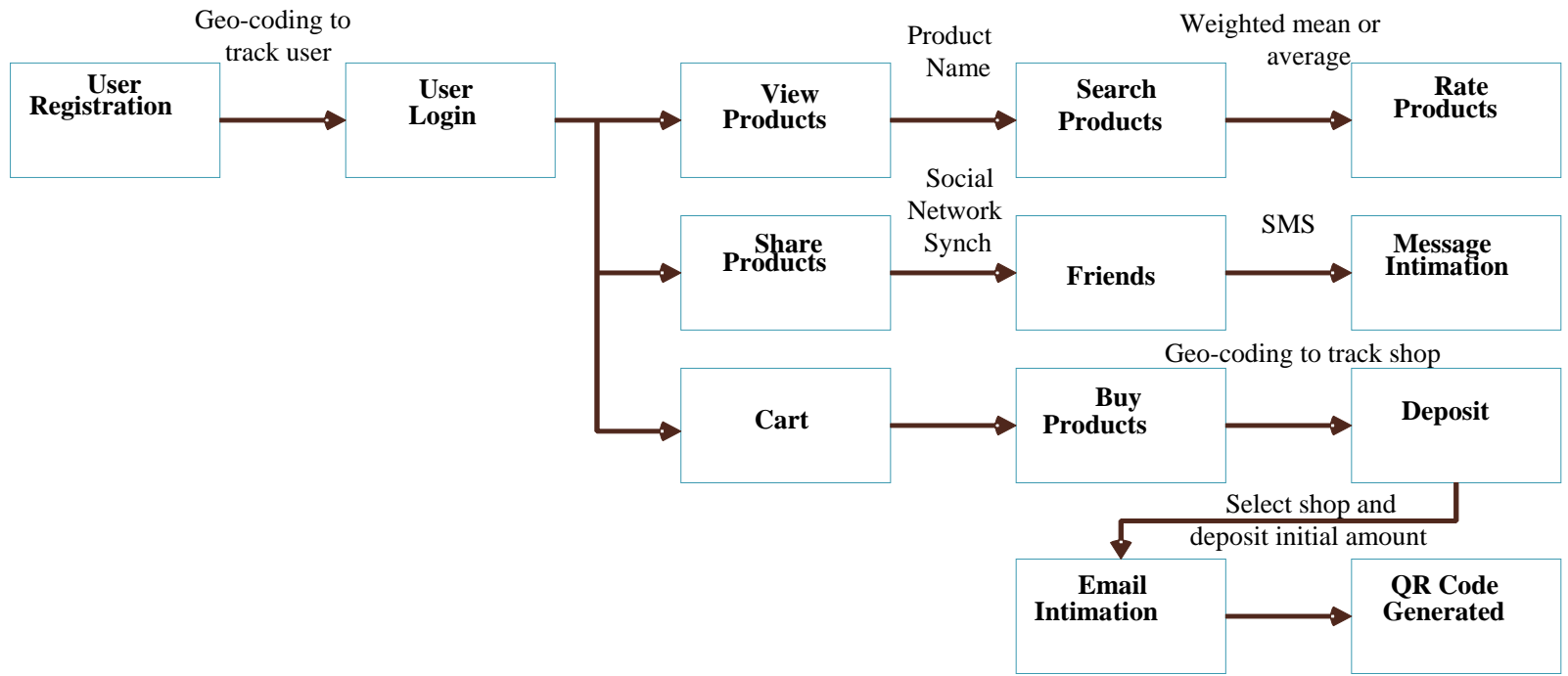

Fig 1: Flow diagram for Customer

\subsubsection{Registration}

The user initially needs to register in promoterApp to utilize the advantages of it. Once the user has registered he/she can login with the registered user name and password. When the user logs in, his current location is retrieved using GPS (Global Positioning System) or network provider. The user is then listed with all the top purchased products. The user can register or login at any time and at any place. While the user logs in his whatsApp contacts are synchronized to the server database. This whatsApp contact is used to share the products and to invite friends to this promoterApp. Similarly, the traders can also register in the promoterApp. They can then post the products that are available in their stores. The product is posted by specifying the product type, name and discount.

\subsubsection{Rating}

The user can rate the products by selecting the stars in the ratings bar. The ratings are gives as follows:

$\begin{array}{ll}* & \text { Poor } \\ * * & \text { Slightly good } \\ * * * & \text { Moderately good } \\ * * * * & \text { Very good }\end{array}$

\section{***** Excellent}

Steps used for Rating:

- $\quad$ Rating displayed as floating point number using ratings star.

- It works on the principle of weighted average of weighted mean.

- Weights are used in terms of stars to compute a decimal value.

Rating calculation is done as follows:

(Sum of weight $*$ number of reviews at that weight) / total number of reviews. An example for rating calculation has been given below:

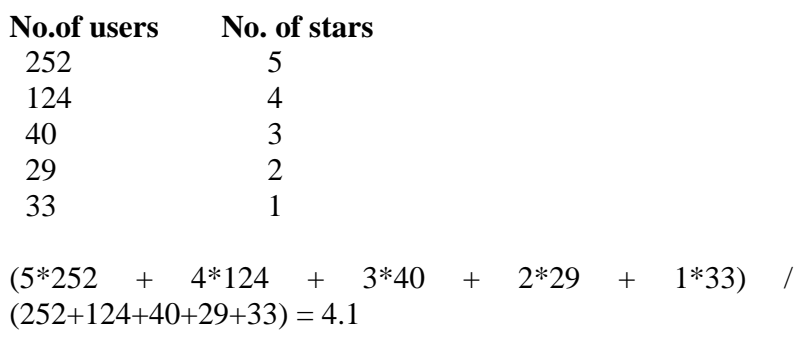

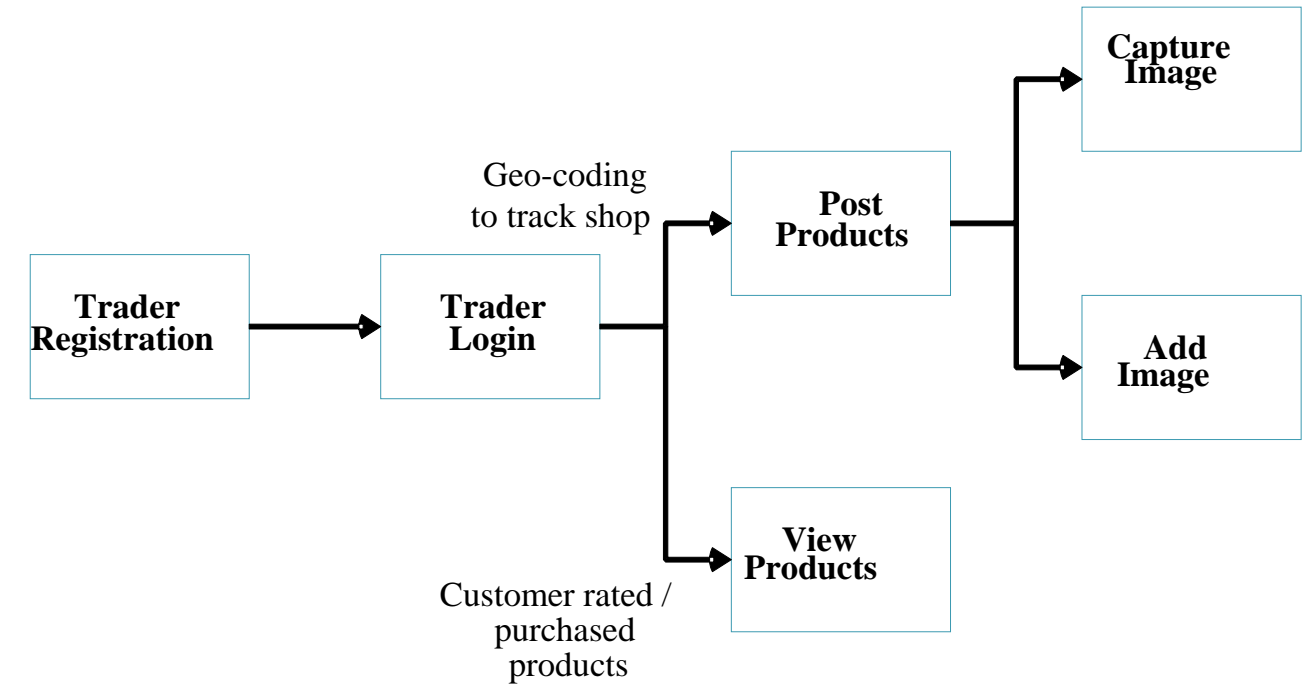

Fig 2: Flow diagram for Trader 
In the above example 252 users have given 5 stars for a product. 124 users have given 4 stars. 40 users have given 3 stars. 29 users have given 2 stars. 33 users have given 1 stars. Total stars are calculated for the product and the total starts are divided by the total number of users rated which will give a weighted average of weighted mean. The product with the highest weighted mean will be the top rated product by the users.

\subsubsection{Search}

The user can search the products either according to the name of the products or based on the type of the products he/she wishes to buy. Search activity receives the query and searches the data, then displays the result. The intent is used to invoke components like start of service, launch activity, displaying a webpage in mobile application API. It is a messaging object that is used for communication with other components. This will search out the required results that the customer requires and returns it to the adapter. The adapter provides access to data items. i.e. it is responsible for displaying the items present in the data set. The results displayed using Customer List View. This list view displays contents and images in the form of list on the product page. The results are either the type or the name of the product that the customer wants to purchase.

\subsubsection{Contact Sync}

The promoterApp uses whatsApp contact for forming groups with respect to a target user. While the user login he/she is asked to synchronize the whatsApp contacts to share the products. Sync Adapter is used to synchronize the contacts with the promoterApp. Sync adapter contains a code to transfer the data (whatsApp contact numbers) between the device and server. Authorization is needed when a data is taken from any of the personal accounts of the user. The promoterApp asks for permission for synchronizing the user's whatsApp contacts. If the user permits, then the promoterApp takes the contacts and stores it in the backend server. When the user grants permission for synchronization a Start Sync activity is received and a thread will be started to run the operation and Perform Sync will be invoked on that thread. All the contacts will be updated in the server. When cancelling sync received matches existing sync operation thread running is interrupted and canceled hence the contacts will not be updated in the server.

\subsubsection{Cart}

If the customer wants to buy any product he can add it to cart. In the cart page the customer can share the product to his friends. The shops that are near to user location which is retrieved from the server database are displayed to him. This uses the technique called geo-coding. Geo-coding is the process of transforming a street address or other description of location into latitude, longitude coordinate. Reverse geocoding is the process of transforming latitude, longitude coordinates into an address. From the list of shops, user can choose a shop. Selection of any shop, by the user in the promoterApp will ask the user to pay some initial amount through credit card number. The promoterApp will also check the validation of the credit card.

Once the shop is selected the email address of the user is asked. An email is sent to the customer along with the QR code and other details about the product. The email address needs to be specified by the user to which the QR code needs to be sent. The QR code is scanned by the trader to find the discount details offer for that user. Each and every time when the user's friends buy the shared product the discount will be updated automatically for all the users who formed a group for that particular product.

\subsubsection{Share}

If the user needs to get the maximum discount for any product he needs to share the products with his friends. When the user's friends purchases the shared product both the user and the user's friend will get the discount, This process gets repeated till the maximum discount is mentioned in that particular product. The user can share the product with his friends either with all the contacts in his friends list or only to specific friends. If the friend the user wants to share is not in this of promoterApp then a message is intimated to his whatsApp number asking him to register in this promoterApp. The shared products can be bought by the users. The first person who shares the product will be taken as a target and grouping takes place with respect to the target.

\subsubsection{QR code}

QR code abbreviated as Quick Response Code, it is a two dimensional bar code. The promoterApp is mainly proposed for discount coupon generation i.e. QR code generation. The $\mathrm{QR}$ code is generated by encrypting the text into dotted images, this is done by DES algorithm and Symmetric key algorithm used for encryption of electronic data. The decryption process is converting dotted image to text format, this is done by converting the small dots in the QR code to binary numbers and validated with an error-correcting technique.

Code words are 8 bits long and use the Reed-Solomon error correction algorithm which is used to detect and correct multiple symbol errors with four error correction levels. The higher the error correction level, the lesser is the storage capacity. In larger QR symbols, the message is broken up into several Reed-Solomon code blocks.

The 4 levels of QR code are:

$$
\begin{array}{ll}
\text { 1. } & \text { Level L (low), } \\
\text { 2. } & \text { Level M (Medium), } \\
\text { 3. } & \text { Level Q (Quartile) and } \\
\text { 4. } & \text { Level H (High). }
\end{array}
$$

The format information records two things: the error correction level and the mask pattern used for the symbol. Masking is used to break up patterns. The message data set is placed from right to left in a zigzag pattern.

\subsection{Implementation Details}

The proposed promoterApp has been developed using Eclipse 4.4.0 Jre 8 as a front end, Wampp as back end and Android 4.3 as platform. Fig. 3 represents the sample screen shots of the promoterApp for posting products used by sellers. The Trader initially needs to register in promoterApp to post the products and to update any requirements. While registration the location of the trader is taken by GPS as latitude and longitude and converted as address and stored in the database. The trader can post the products in this application, using capture and can post the product by specifying the title of the product, type of the product and also the maximum discount of the product. After specifying the details of the product the trader can capture the photo of the product using capture button or the trader can also add the image from the gallery using add image button and then he can directly post the product into the promoterApp. The trader can cancel his post using cancel option. After posting the product that product will automatically be updated into the promoter application and the users can easily search that product specifying either 
the type or name of the product. Fig. 4 represents sharing products with friends used by buyers.

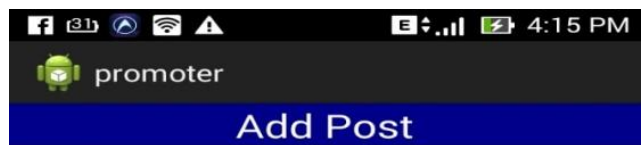

\begin{tabular}{l} 
Ad title vestar \\
air conditioner \\
\hline 25 \\
\hline
\end{tabular}
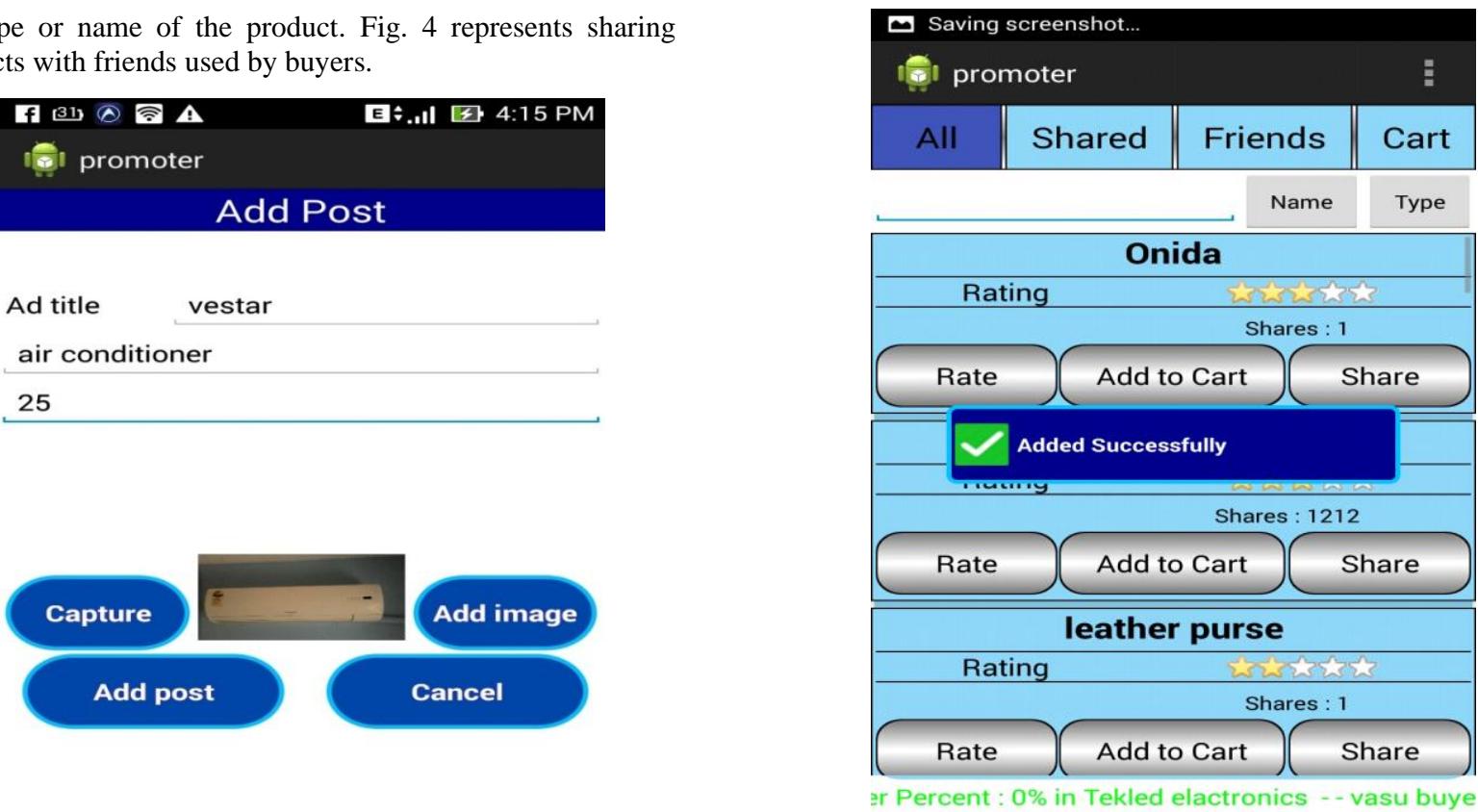

Fig 4: Product Share Page

Fig 3: Products Post Page

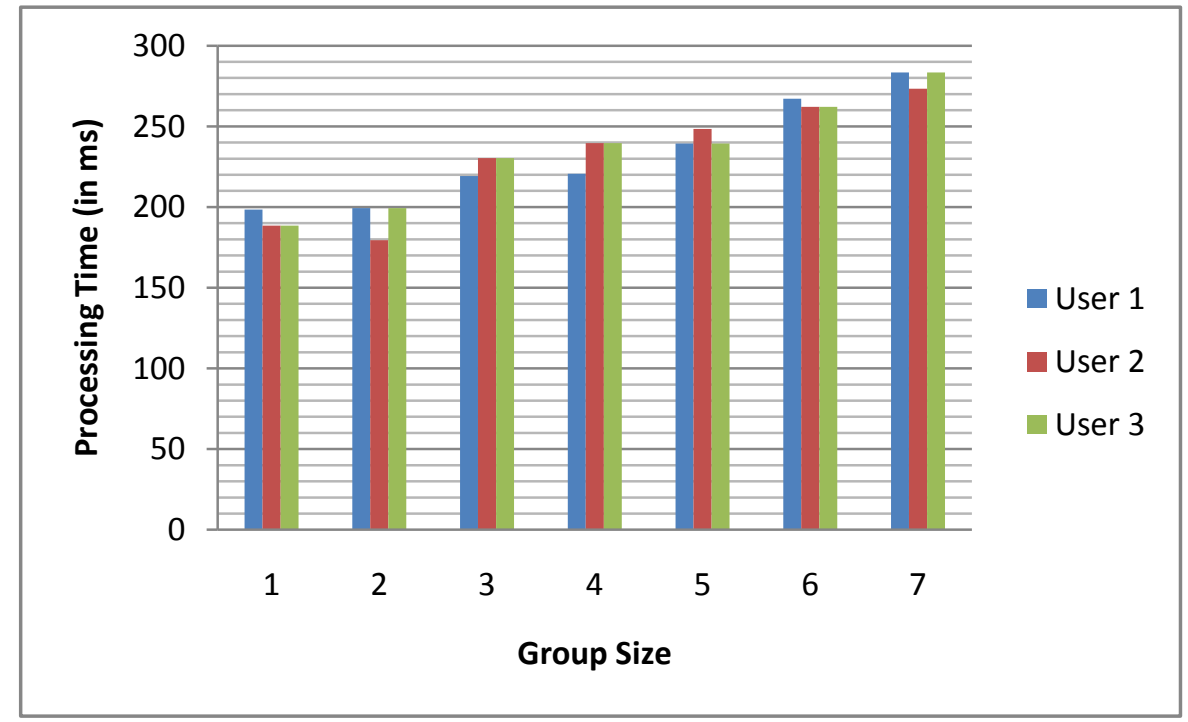

Fig 5: Average processing time of promoterApp for generating product recommendations for groups

\section{EVALUATION}

To assess the efficiency of promoterApp, 50 groups for a given user has been considered. For evaluation purpose a predefined size of 2 to 8 for each group has been taken. The average processing time of promoterApp is considered in generating recommendations for each one of the 50 groups. From the Fig. 5 it has been inferred that the average processing time (in milliseconds) required by promoterApp to generate group recommendations does not exponentially increase when the number of group members increases. Instead, as determined by the curve created using the Microsoft Excel Trend tool also shown in Fig. 5, the increase in processing time of promoterApp when the number of group members increases follows a linear trend, which demonstrates the scalability of the promoterApp.
Based on the top $\mathrm{N}$ recommendations provided, the effectiveness of the proposed approach were analyzed using precision, recall and F1 measures. Precision is the fraction of retrieved instances that are relevant, recall is the fraction of relevant instances that are retrieved and F1 score can be interpreted as a weighted average of the precision and recall. The results obtained for the top 5 recommended products for 50 users has been listed in the Table 2 and Table 3 for the existing work[9] and proposed work. In Table 4 the average values for precision, recall and F1 measures have been provided. From the Table 4 it has been observed that the proposed work i.e the pomoterApp performs better compared to the recommendation provided by the existing work. 
Table 2. Experimental results for existing work

\begin{tabular}{|c|c|c|c|c|c|c|c|c|c|c|c|}
\hline Measures & $\mathbf{u 1}$ & $\mathbf{u 2}$ & $\mathbf{u 3}$ & $\mathbf{u 4}$ & $\mathbf{u 5}$ & $\mathbf{u 6}$ & $\mathbf{u 7}$ & $\mathbf{u 8}$ & $\mathbf{u 9}$ & $\cdots$ & $\mathbf{u 5 0}$ \\
\hline Precision & 0.0694 & 0.0884 & 0.0842 & 0.0653 & 0.0442 & 0.0751 & 0.0633 & 0.0744 & 0.0120 & $\ldots$ & 0.0533 \\
\hline Recall & 0.0255 & 0.0234 & 0.0443 & 0.0148 & 0.0186 & 0.0180 & 0.0111 & 0.0313 & 0.0219 & $\ldots$ & 0.0255 \\
\hline F1measure & 0.0372 & 0.0370 & 0.0580 & 0.0241 & 0.0261 & 0.0290 & 0.0188 & 0.0442 & 0.0155 & $\ldots$ & 0.0344 \\
\hline
\end{tabular}

Table 3. Experimental results for proposed work

\begin{tabular}{|c|c|c|c|c|c|c|c|c|c|c|c|}
\hline Measures & $\mathbf{u 1}$ & $\mathbf{u 2}$ & $\mathbf{u 3}$ & $\mathbf{u 4}$ & $\mathbf{u 5}$ & $\mathbf{u 6}$ & $\mathbf{u 7}$ & $\mathbf{u 8}$ & $\mathbf{u 9}$ & $\ldots$ & $\mathbf{u 5 0}$ \\
\hline Precision & 0.1650 & 0.1288 & 0.1200 & 0.1600 & 0.1202 & 0.0418 & 0.1200 & 0.1600 & 0.1608 & $\ldots$ & 0.1233 \\
\hline Recall & 0.0615 & 0.0830 & 0.0500 & 0.0400 & 0.0756 & 0.0590 & 0.0111 & 0.0723 & 0.0533 & $\ldots$ & 0.0665 \\
\hline F1measure & 0.0896 & 0.1009 & 0.0705 & 0.0641 & 0.0928 & 0.0489 & 0.0203 & 0.0995 & 0.0800 & $\ldots$ & 0.0864 \\
\hline
\end{tabular}

Table 4. Average values for precision, recall and F1 measure for Top 5 recommendations

\begin{tabular}{|c|c|c|}
\hline Measures & Existing work & Proposed Work \\
\hline Precision & 0.0629 & 0.1299 \\
\hline Recall & 0.0234 & 0.0572 \\
\hline F1 Measure & 0.0324 & 0.0753 \\
\hline
\end{tabular}

\section{CONCLUSION}

Existing recommendation systems provide personalized recommendations for individual customers. But in the case of activities performed by a group of individuals these systems could not provide group recommendations. In this project a recommendation system for generating group coupon has been proposed. Based on the proposed approach a mobile application called promoterApp has been developed for mobile users in order to generate a group discount coupon i.e QR code. It mainly recommends location based products for the customers. From the experiments conducted it has been observed that recommending products with a discount for a group purchase could significantly increase the customer willingness to purchase. Furthermore the proposed groupdiscount mechanism could effectively assist traders to increase their revenues by attracting the maximum number of group members through taking advantage of the power of social influence, and location sensitivity. This application does not take the user preference and recommend the products, but it keeps track of top purchased and top rated products and recommends to all the users. This application can be enhanced by keeping track of the ratings given by the user to each product and his preference is found by calculating top rated product types or brand and then he is recommended products according to his preference.

\section{REFERENCES}

[1] Chen, Y.L., Cheng, L.C. and Chuang, C.N., 2008. A group recommendation system with consideration of interactions among group members. Expert systems with applications, 34(3), pp.2082-2090

[2] Kim, J.K., Kim, H.K., Oh, H.Y. and Ryu, Y.U., 2010. A group recommendation system for online communities. International Journal of Information Management, 30(3), pp.212-219.
[3] Park, M.H., Hong, J.H. and Cho, S.B., 2007. Locationbased recommendation system using bayesian user's preference model in mobile devices. In Ubiquitous Intelligence and Computing (pp. 1130-1139). Springer Berlin Heidelberg.

[4] Pera, M.S. and Ng, Y.K., 2013. A group recommender for movies based on content similarity and popularity. Information Processing \& Management,49(3), pp.673-687.

[5] Baatarjav, E.A., Phithakkitnukoon, S. and Dantu, R., 2008, November. Group recommendation system for facebook. In On the Move to Meaningful Internet Systems: OTM 2008 Workshops (pp. 211-219). Springer Berlin Heidelberg.

[6] Bobadilla, J., Ortega, F., Hernando, A. and Gutiérrez, A., 2013. Recommender systems survey. Knowledge-Based Systems, 46, pp.109-132.

[7] Kim, J.K., Kim, H.K., Oh, H.Y. and Ryu, Y.U., 2010. A group recommendation system for online communities. International Journal of Information Management, 30(3), pp.212-219.

[8] Gediminas Adomavicius and Alexander Tuzhilin. Toward the next generation of recommender systems: A survey of the state-of-the-art and possible extensions. IEEE Transactions on Knowledge and Data Engineering, 17(6):734-749, June 2005.

[9] Contreras, D., Salamó, M. and Pascual, J., 2015. A WebBased Environment to Support Online and Collaborative Group Recommendation Scenarios. Applied Artificial Intelligence, 29(5), pp.480-499.

[10] Christensen, I., Schiaffino, S. and Armentano, M., 2016. Social group recommendation in the tourism domain. Journal of Intelligent Information Systems, pp.1-23. 